\title{
Gamma-ray families with halos: Main characteristics and possibilities of using them to estimate the $p+H e$ fraction in the mass composition of cosmic rays at energies $1-100 \mathrm{PeV}$
}

\author{
A.S. Borisov ${ }^{1}$, V.G. Denisova ${ }^{1}$, Z.M. Guseva ${ }^{1}$, E.A. Kanevskaya ${ }^{1}$, S.E. Pyatovsky ${ }^{1}$,, M.G. Kogan ${ }^{1}$, A.E. Morozov ${ }^{1}$ \\ R.A. Mukhamedshin ${ }^{2}$, V.S. Puchkov ${ }^{1}$, S.B. Shaulov ${ }^{1}$, G.P. Shoziyoev ${ }^{1}$, and M.D. Smirnova ${ }^{1}$ \\ ${ }^{1}$ P.N. Lebedev Physical Institute of the Russian Academy of Sciences, Moscow, Russia \\ ${ }^{2}$ Institute for Nuclear Research, Russian Academy of Sciences, Moscow, Russia

\begin{abstract}
Characteristics of $\gamma$-ray families with halos (XREC, Pamir) and data of experiments with EAS are analyzed to estimate the proton and helium $(\mathrm{p}+\mathrm{He})$ fractions in the primary cosmic radiation at $E_{0}=1-100 \mathrm{PeV}$. It is shown that at energies $E_{0} \sim 1-100 \mathrm{PeV}$ the fraction of p+He remains significant, namely, the fraction of $\mathrm{p}+\mathrm{He}$ is near $40 \%$ at $E_{0}=10 \mathrm{PeV}$.
\end{abstract}

\section{XREC (Pamir) experiment}

The X-ray emulsion chamber (XREC) (Pamir) (Fig. 1) represents a solid-state track chamber installed at the Pamirs at an altitude of $4400 \mathrm{~m}$ a.s.l. The air above the chamber is a thick target $\left(600 \mathrm{~g} / \mathrm{cm}^{2}\right)$ where nuclearelectromagnetic cascades (NEC) induced by particles of the primary cosmic radiation (PCR) take place. Single particles of the high-energy electromagnetic component ( $\gamma$ and $e^{ \pm}$called below for brevity " $\gamma$-rays") of the air cascades initiate electron-photon cascades $(\mathrm{EPhC})$ in the upper lead plates of the chamber, assembled in a stack and called $\Gamma$-block. These $\mathrm{EPhCs}$ are recorded as dark spots by X-ray films placed between and under lead plates if the energy of incident $\gamma$-rays and electrons/positrons is high enough $\left(E_{0} \geq 4 \mathrm{TeV}\right)$.

Processing of the experimental data was performed using the MCO [1] and QGSJ models. The MC0 model well describes the XREC experimental data and corresponds to LHC data in the low energy region. The MC0 model designed for simulation of hadron-hadron and hadron-nucleus interactions in Extensive Air Showers (EAS) reproduces rather well the characteristics of the XREC experimental data and high- $x_{F}$ LHC data.

Analysis of XREC (Pamir) experiment results using MC0 and QGSJ models shows that $\geq 90 \%$ of the registered events are formed by the $\mathrm{p}+\mathrm{He}$ component.

\section{2. $\gamma$-ray families with halos in XREC (Pamir)}

In $\gamma$-ray families with energies $\Sigma E_{\gamma} \geq 500 \mathrm{TeV}, \gamma$-rayinitiated $\mathrm{EPhCs}$ overlap, creating regions with high optical density in the centers of these $\gamma$-ray families (Fig. 2). As follows from Fig. 3, simulations explain the nature of $\gamma$-ray families with halos in the framework of the traditional interaction models (MC0 and QGSJ).

a e-mail: sep@lebedev.ru
To study the problem of halos, $\gamma$-ray families were simulated in the range of PCR particle energies $E_{0}=0.3-3000 \mathrm{PeV}$ under the following selection criteria:

- minimum energy of $\gamma$-ray families is $100 \mathrm{TeV}$;

- minimum number of particles in $\gamma$-ray families is $N_{\text {chmin }}=3$

- PCR particles are presented by nine groups of nuclei, namely, p, He, $\mathrm{Li}, \mathrm{C}, \mathrm{O}, \mathrm{Mg}, \mathrm{Si}, \mathrm{V}, \mathrm{Fe}$;

- the observation level is $594 \mathrm{~g} / \mathrm{cm}^{2}$ for XREC (the Pamirs) and $695 \mathrm{~g} / \mathrm{cm}^{2}$ for EAS+XREC HADRON experiment (the Tian Shan);

- the threshold energy is $100 \mathrm{GeV}$ for $\gamma$-rays and stable hadrons arriving on the top of the XREC from the air;

- the zenith angle varies from 0 to 0.9 radian.

Simulations of halos were previously done using simulated $\gamma$-ray families with the use of a Monte-Carlo program which applies a "chessboard" algorithm. A preliminary selection was made which was consistent with the criteria for processing experimental XREC data:

- only $e^{ \pm}$and $\gamma$-rays are selected;

- $\gamma$-ray families with energy $\Sigma E_{\gamma} \geq 100 \mathrm{TeV}$ and average distance of particles from the center $\mathrm{R} \leq 15 \mathrm{~cm}$ are used.

The "chessboard" algorithm of the halos calculation applies Spatial Distribution Functions (SDF) of $e^{ \pm}$and $\gamma$-rays. The SDFs are calculated by the model simulation for XREC (Pamir) with the configuration shown in Fig. 1. The SDF considers the distance of a $\gamma$-ray $(\mathrm{R}, \mathrm{cm})$ from the cascade core, the depth of the cascade development (T, rad.unit), $E_{e^{ \pm}}(\mathrm{MeV})$ and the probability that the first interaction will occur at a certain depth in XREC.

Figure 2 demonstrates examples of a scanned image of the experimental "FIANIT" halo event (left) and calculated halo formed by the primary proton $\left(E_{p}=420 \mathrm{PeV}\right)$ (right) with close halo areas, $S_{\text {halo }}$, of $1020 \mathrm{~mm}^{2}$ and $2100 \mathrm{~mm}^{2}$, respectively. 


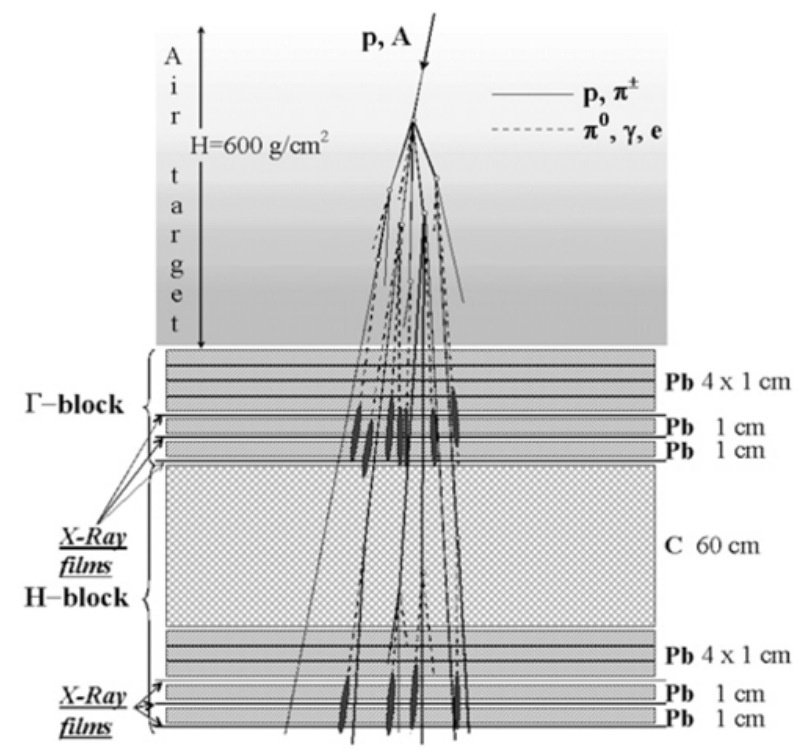

Figure 1. Layout of the Pamir experiment setup.
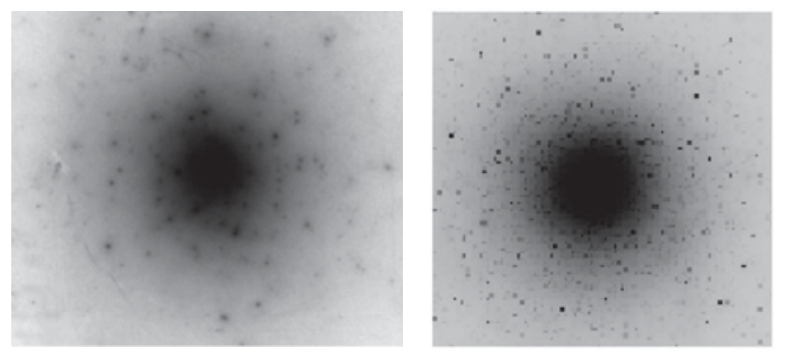

Figure 2. Scanned image of the «FIANIT »halo event $\left(E_{0} \sim\right.$ $400 \mathrm{PeV}, \mathrm{S}_{\text {halo }}=1020 \mathrm{~mm}^{2}$ ) (left); calculated halo formed by the primary proton $\left(E_{p}=420 \mathrm{PeV}, \mathrm{S}_{\text {halo }}=2100 \mathrm{~mm}^{2}\right)$ (right).

The criteria for selection of the $\gamma$-ray families with halos in the XREC (Pamir) experiment are as follows $[2,3]$ :

- $\Sigma E_{\gamma} \geq 500 \mathrm{TeV}$;

- an area of halo $S_{D=0.5}$ bounded by isodense with optical density $\mathrm{D}=0.5$.

- $S_{D=0.5} \geq 4 \mathrm{~mm}^{2}$ - for single-center halo;

- $\Sigma S_{D=0.5} \geq 4 \mathrm{~mm}^{2}$, if $S_{D=0.5} \geq 1 \mathrm{~mm}^{2}$-for a multicenter halo.

Model calculations show that the $\gamma$-families with $\Sigma E_{\gamma}=$ $100-400 \mathrm{TeV}\left(E_{\gamma} \geq 4 \mathrm{TeV}\right)$ are produced mainly by PCR particles (protons) with $E_{0}=1-10 \mathrm{PeV}$, while the halo $\gamma$-families with $\Sigma E_{\gamma} \geq 500 \mathrm{TeV}$ are produced by PCR particles with $E_{0} \geq 10 \mathrm{PeV}$.

The statistics of the Pamir experiment includes 61 $\gamma$-ray families with halos at ST $=3000 \mathrm{~m}^{2}$. year.sr.

Calculations have shown that $\gamma$-ray families with halo are created as a result of the overlapping of SDFs of EPhC initiated by $e^{ \pm}$and $\gamma$-rays. Therefore, the halo is not some exotic phenomenon but the central part of a high energy EAS.

The analysis of $\gamma$-ray families with halos allows us to apply these events for estimating the PCR mass composition because the halo phenomenon observed in XREC experiments is predominantly formed by protons and helium nuclei $(\geq 90 \%)$. Its origin is in the SDF

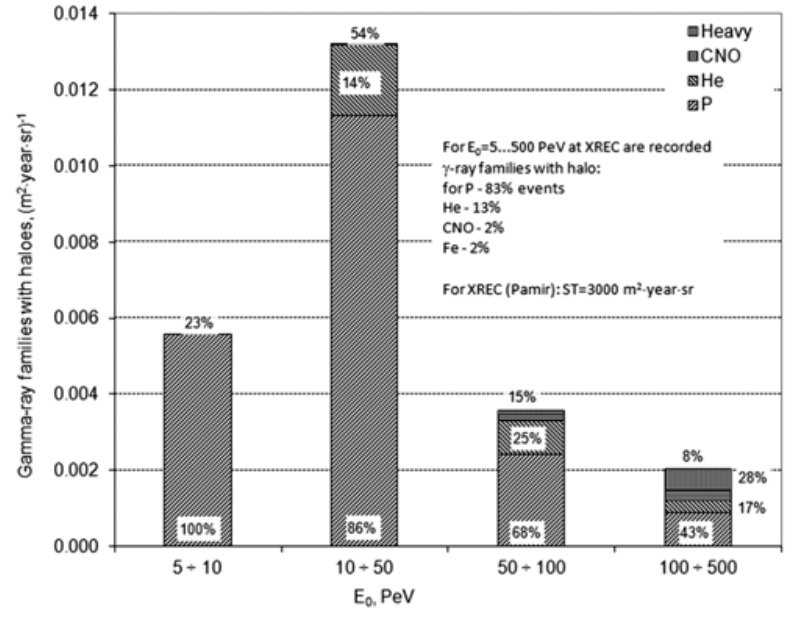

Figure 3. Dependence of the intensity of $\gamma$-ray families with halos and relative fractions of different PCR nuclei producing these families on $E_{0}$.

Table 1. The mass composition in the MC0 model.

\begin{tabular}{|l|l|l|l|}
\hline E PeV & 1 & 10 & 100 \\
\hline$p \%$ & 33 & 26 & 20 \\
\hline $\mathrm{He} \%$ & 22 & 17 & 15 \\
\hline
\end{tabular}

overlapping of EPhC initiated by $e^{ \pm}$and $\gamma$-rays and is almost independent of the simulation model.

\section{Dependence on $\mathrm{E}_{0}$ of the fraction of $\gamma$-ray families with halos}

The dominant part of the halo-containing $\gamma$-ray families is formed by protons (Fig. 3). Observation of a large number of such events indicates a notable presence of protons in the PCR spectrum up to $E_{0} \sim 100 \mathrm{PeV}$.

Model calculations show that when the PCR consists of protons only, the number of observed halos should be equal to 140 instead of 61 registered by the Pamir experiment, 34 and 5 in the cases for pure helium and heavy nucleus spectra, respectively. It follows from the above that the fractions of PCR protons and helium nuclei are higher than $15 \%$ and $24 \%$, respectively. The summary $\mathrm{p}+\mathrm{He}$ fraction is higher than $39 \%$ at $E_{0}=10 \mathrm{PeV}$.

The mass composition in MC0 model is presented in Table 1. Calculations revealed that $83 \%$ of all events are produced by primary protons, $13 \%$ are produced by helium nuclei and not more than $4 \%$ are produced by heavier nuclei. This conclusion is almost independent of the model and thus provides a possibility to estimate the proton fraction in PCR in the range of $E_{0}=1-100 \mathrm{PeV}$.

\section{Fractions of $\gamma$-ray families with multicenter halos generated by different nuclei}

In some cases the halo consists of several centers. The multicenter of a halo is a parameter which strongly depends on the type of primary nuclei (Table 2).

It is evident that recorded $\gamma$-ray families with one halo are almost entirely generated by primary protons with a possible small addition of He. This conclusion 


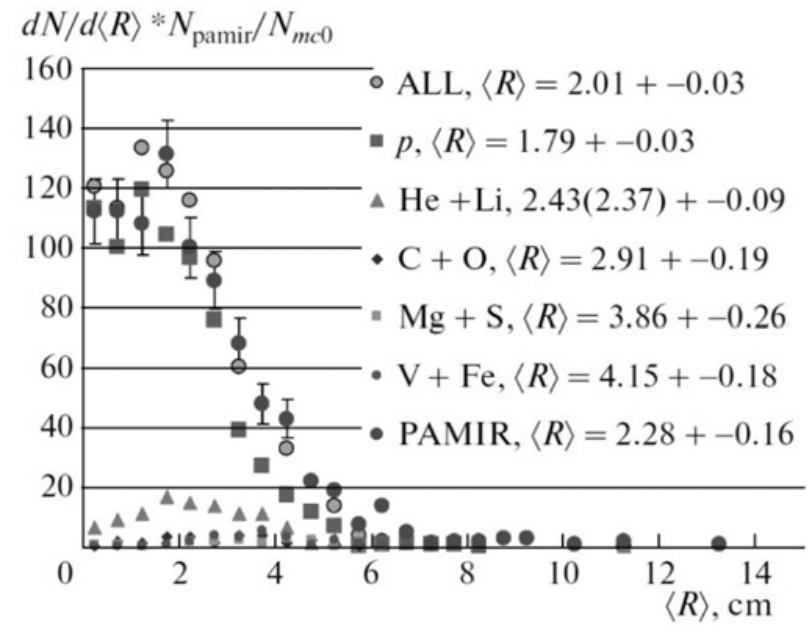

Figure 4. Distribution over average size of the $\gamma$-ray families, $\mathrm{dN} / \mathrm{d}\langle\mathrm{R}\rangle$. Simulated distributions for $\mathrm{MC} 0$ are normalized to the number of experimental $\gamma$-ray families observed in the XREC (Pamir) experiment.

Table 2. Fraction of $\gamma$-ray families with multicenter halos generated by different nuclei.

\begin{tabular}{|l|c|c|c|c|c|}
\hline $\mathrm{p}$ & $\mathrm{He}$ & $\mathrm{C}$ & $\mathrm{Fe}$ & $\mathrm{MC} 0$ & PAMIR \\
\hline 0.25 & 0.45 & 0.59 & 0.70 & 0.28 & 0.23 \\
\pm 0.03 & \pm 0.09 & \pm 0.11 & \pm 0.03 & \pm 0.03 & \pm 0.07 \\
\hline
\end{tabular}

Table 3. The probabilities of halo appearance.

\begin{tabular}{|l|c|c|}
\hline $\mathrm{p}$ & $\mathrm{He}$ & $>\mathrm{He}$ \\
\hline $1.76 \%$ & $0.44 \%$ & $0.13 \%$ \\
\hline
\end{tabular}

is confirmed by the probabilities of halo appearance (Table 3).

MC0-based calculations show that at $E_{0}>5 \mathrm{PeV}$ the efficiency of formation of the halo-containing $\gamma$-ray families by protons is four times higher than by He nuclei (Table 3). This explains the preferential registration with XREC of such $\gamma$-ray families produced by protons.

\section{Average size of $\gamma$-ray families in the XREC experiment}

According to calculations [2,3], the average size $\langle R\rangle$ of the $\gamma$-ray families formed by protons is indeed $1.8 \mathrm{~cm} ;\langle\mathrm{R}\rangle=$ $4.5 \mathrm{~cm}$ for events formed by iron nuclei. Experimental and calculated $\langle\mathrm{R}\rangle$ distributions are shown in Fig. 4. $\gamma$-ray families with energy $\Sigma E_{\gamma}=100-400 \mathrm{TeV}$, where reliable identification of all cascades is possible, were used to construct these distributions. At high family energies, a halo can occur in the central part of an event; single low-energy cascades, initiated by $\gamma$-rays in lead, can be lost inside these halos, and the determination of $\langle\mathrm{R}\rangle$ can be complicated. It can be seen from Fig. 4 that the experimental $\langle\mathrm{R}\rangle$ distribution is well described by the set of spatial distributions of events formed by primary protons. The average size of $\gamma$-ray families in the XREC of the Pamir experiment is $\left\langle R_{\text {Pamir }}\right\rangle=2.28 \mathrm{~cm}$, which is somewhat higher than that predicted by the MC0 model, i.e., $\left\langle R_{M C 0}\right\rangle=2.01 \mathrm{~cm}$, and virtually coincides with the

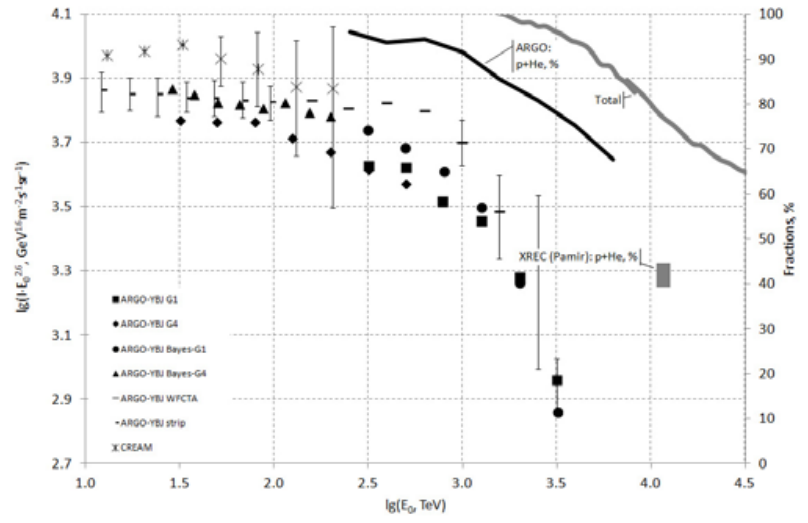

Figure 5. The $\mathrm{p}+\mathrm{He}$ fraction according to the ARGO, CREAM and XREC (Pamir) experiments depending on $E_{0}$.

average size of events formed by $\mathrm{He},\left\langle R_{\mathrm{He}}\right\rangle=2.37 \mathrm{~cm}$ (Fig. 4).

An analysis of XREC response shows that the spatial distribution of cascades in the $\gamma$-ray families as it passes through XREC increases by $15 \%$. The overestimation of $\langle R\rangle$ in the experiment is associated with the registration due to fluctuations of a large number of families with energies close to the threshold energy $\left(\Sigma E_{\gamma t h r}=100 \mathrm{TeV}\right)$. Such families have a broader spatial distributions. The true size of experimental $\gamma$-ray families is $\left\langle R_{\text {Pamir }}\right\rangle=(1.94 \pm 0.06) \mathrm{cm}$, which proves the above made conclusion on the leading role of primary protons in the formation of $\gamma$-ray families registered in the XREC.

\section{Proton+helium fraction at $E_{0}=1-100 \mathrm{PeV}$ according to the ARGO, CREAM and XREC (Pamir) experiments}

The p+He fraction at $E_{0}=1-100 \mathrm{PeV}$ according to the ARGO, CREAM [4,5] and XREC (Pamir) experiments depending on $E_{0}$ is shown in Fig. 5.

The estimate of the $\mathrm{p}+\mathrm{He}$ fraction found in the XREC (Pamir) experiment is based on the analysis of the flow of $\gamma$-ray families with halos and known probabilities of formation of halos by protons, helium and heavier nuclei.

The experimental number of the $\gamma$-ray families with halos (61) is compared with the expected number of halos created by protons, helium and heavier nuclei. It is shown, that if the PCR would consist of only protons, the number of halos must be equal to 140 . In the case of dominance of helium or heavier nuclei, the number of halos must be equal to 34 or 5 , respectively. Thus we conclude there is a significant fraction of protons in the PCR on the basis of the experimental number of $\gamma$-ray families with halo.

To explain the observed number of $\gamma$-ray families with halos in the XREC (Pamir) experiment, the $\mathrm{p}+\mathrm{He}$ fraction should be higher than $39 \%$.

\section{Conclusions}

1. Our calculation explains the nature of $\gamma$-ray families with halos in the framework of a traditional model of hadron interactions, which gives a rather good description of the XREC data. 
2. According to the XREC (Pamir) and HADRON (Tian Shan) experiments the $\mathrm{p}+\mathrm{He}$ component is noticeably present at $E_{0}=1-100 \mathrm{PeV}$.

3. Fractions of protons and He nuclei in the PCR mass composition at $E_{0}=10 \mathrm{PeV}$ are as follows:

- protons - higher than $15 \%$

- $\mathrm{p}+\mathrm{He}$ - higher than $39 \%$.

\section{References}

[1] G.F. Fedorova, R. A. Mukhamedshin. Bull. Soc. Sci. Lett. Lodz, Ser. Rech. Def., XVI, 137 (1994)

[2] V.S. Puchkov, A.S. Borisov, Z.M. Guseva, V.G. Denisova, E.A. Kanevskaya, M.G. Kogan,
V.M. Maximenko, A.E. Morozov, R.A. Mukhamedshin, S.E. Pyatovsky, M.D. Smirnova. Bulletin of the Russian Academy of Sciences Physics, 2011, 75(3), pp. 392-394

[3] V.S. Puchkov, A.S. Borisov, Z.M. Guseva, V.G. Denisova, E.A. Kanevskaya, M.G. Kogan, V.M. Maximenko, A.E. Morozov, R.A. Mukhamedshin, S.E. Pyatovsky, M.D. Smirnova. 32nd International Cosmic Ray Conference, Beijing 2011, HE1.2

[4] Zhang S.S., Cao Z. 34th International Cosmic Ray Conference, 30 July - 6 August - 2015 - Hague, Netherlands. PoS (ICRC2015) 261

[5] I. De Mitri on behalf of the ARGO-YBJ collaboration. EPJ Web of Conferences 99, 08003 (2015). doi: 10.1051/epjconf/20159908003 